Vol. 23, No. 4, pp. 350 357, 2020.

\title{
Computational and Experimental Study on Electronic Band Structure of Bismuth Ferrite: A Promising Visible Light Photocatalyst
}

\author{
G Thamizharasan', Eithiraj R.D², Enhbayar Enhtuwshin ${ }^{3}$, So Jung Kim³, \\ Niroj Kumar Sahu ${ }^{4}$, Arpan Kumar Nayak ${ }^{1}$, HyukSu Han ${ }^{3 \dagger}$
}

\footnotetext{
${ }^{1}$ Department of Physics, School of Advanced Sciences, Vellore Institute of Technology, Vellore 632014, India ${ }^{2}$ Department of Physics, Computational Material Science Lab, School of Advanced Sciences, Vellore Institute of Technology, Vellore 632014, India

${ }^{3}$ Department of Energy Engineering, Konkuk University, 120 Neungdong-ro, Gwangjin-gu, Seoul 05029, Republic of Korea ${ }^{4}$ Centre for Nanotechnology, Vellore Institute of Technology, Vellore 632014, India
}

(Received December 8, 2020; Revised December 8, 2020; Accepted December 9, 2020)

\begin{abstract}
s
Electronic band structure of bismuth ferrite (BFO) is studied by computational and experimental methods. Bandgap of $\mathrm{BFO}$ is precisely determined using optical absorption spectra as well as density functional calculation (DFT). Both methods give a comparable result that BFO can have both of direct or indirect (very close to direct) bandgap of about 2.0 2.2 eV. Furthermore, electronic transition in BFO occurs via the unoccupied $\mathrm{O} 2 \mathrm{p}$ to the occupied $\mathrm{Fe} 3 \mathrm{~d}$ states or the $\mathrm{d}-\mathrm{d}$ transition in $\mathrm{Fe} 3 \mathrm{~d}$ states. Intriguing electronic structure of BFO, a narrow bandgap and a multiple electronic transition route, render it as a promising candidate for a visible light photocatalyst.
\end{abstract}

Keywords: Bismuth ferrite, semiconductor, photocatalyst, water splitting

\section{Introduction}

Photoelectrochemical water platting is a one of promising way to produce sustainable energy without forming any greenhouse gases. ${ }^{[1]}$ The solar energy can be directly converted to chemical energy such as hydrogen $\left(\mathrm{H}_{2}\right)$ by photocatalysis of water. Semiconductor metal oxides have

${ }^{\dagger}$ Corresponding Author: HyukSu Han

E-mail: hhan@konkuk.ac.kr 
been widely studied as an active photoanode for photoelectrochemical water splitting due to their optimal band structure and electrochemical robustness in harsh electrolyte conditions. ${ }^{[2]}$ Generally, a narrow bandgap with an appropriate band positon is favorable for semiconductor metal oxides to effectively absorb visible light and split water.

Bismuth ferrite $\left(\mathrm{BiFeO}_{3}, \mathrm{BFO}\right)$ has attracted a great attention from researchers owing to attractive electronic and magnetic properties. ${ }^{[3,4]}$ It was reported that $\mathrm{BFO}$ has a narrow direct bandgap of around $2.2 \mathrm{eV}$ and is responsive to visible-light for photocatalytic water splitting. ${ }^{[5]}$ However, several discrepancies were found in the literature regarding with the electronic band structure of BFO, especially between computational and experimental results. ${ }^{[6,7]}$ Difference values for the bandgap of $\mathrm{BFO}$ were reported. ${ }^{[8]}$ Moreover, although the ferroelectric properties of $\mathrm{BFO}$ with the polarizable electronic configuration of $\mathrm{Bi}^{3+}$ can enhance its photocatalytic activity, this gives difficulty to experimentally study a detailed electronic band structure of BFO. Thus, combined approach using computational as well as experimental methods is necessary to accurately investigate the electronic band structure of BFO.

In this work, we have prepared $\mathrm{BFO}$ via a facile sol-gel method and analyze its optical and electronic properties by measuring absorption spectra. Also, computational simulations were performed on the modeled $\mathrm{BFO}$ to precisely calculate electronic band structure of BFO. Our results reveal that $\mathrm{BFO}$ can have a direct or indirect (very close to direct) bandgap with a value of about $2.0 \sim 2.2 \mathrm{eV}$. Moreover, electronic transitions in $\mathrm{BFO}$ can be occurred via a two different routes either from the occupied $\mathrm{O} 2 \mathrm{P}$ to the unoccupied $\mathrm{F} 3 \mathrm{~d}$ states or the $\mathrm{d}-\mathrm{d}$ transition in $\mathrm{Fe}$ 3d states. Experimental and computational results for electronic structure of $\mathrm{BFO}$ were in good agreement each other. A narrow bandgap of $\mathrm{BFO}$ with a multiple electronic transition route highlights a potential use of $\mathrm{BFO}$ as an active visible light photocatalyst for water splitting.

\section{Experimental Procedures}

Synthesis and characterization: The BFO was synthesized using a facile sol-gel route. Typically, $\mathrm{Bi}\left(\mathrm{NO}_{3}\right)_{3} \cdot 6 \mathrm{H}_{2} \mathrm{O}$ and $\mathrm{Fe}\left(\mathrm{NO}_{3}\right)_{3} \cdot 9 \mathrm{H}_{2} \mathrm{O}$ in a stoichiometry ratio (1:2) were dissolved in ethylene glycol by continuous stirring at $80^{\circ} \mathrm{C}$ for $30 \mathrm{~min}$. Then the reaction was carried out at $70^{\circ} \mathrm{C}$ for 10 $\mathrm{h}$ to get a gel. Then gel was heated in a muffle furnace at $400^{\circ} \mathrm{C}$ for $2 \mathrm{~h}$ followed by annealing at $550^{\circ} \mathrm{C}$ for $1 \mathrm{hr}$ in a ramp rate $5^{\circ} \mathrm{C} / \mathrm{min}$. Finally the powder was collected by repeated washing with ethanol and water and dried at $60^{\circ} \mathrm{C}$ for overnight. Crystal structure of the $\mathrm{BiFeO}_{3}$ were refined from powder X-ray diffractometer (Malvern Panalytical $\mathrm{X}^{\text {'Pert }}{ }^{3}$ Powder).We used Fullprof as a refinement tool for XRD patterns. Chi-square value as set to 2.75. In addition, these refined structures optimized by the wien2k 2D optimization code. The UV-vis spectrum of BFO powder was recorded using a JASCO UV Visible Spectrophotometer (V670 PC).

Computational methods: First principles calculation of ferromagnetic $\mathrm{R} 3 \mathrm{c} \mathrm{BiFeO} 3$ executed using full potential linearized augmented plane wave (FP-LAPW) method. This density functional theory (DFT) ground state calculations performed 


\section{특 집 ‥ G Thamizharasan, Eithiraj R.D, Enhbayar Enhtuwshin, So Jung Kim, Niroj Kumar Sahu, Arpan Kumar Nayak, HyukSu Han}
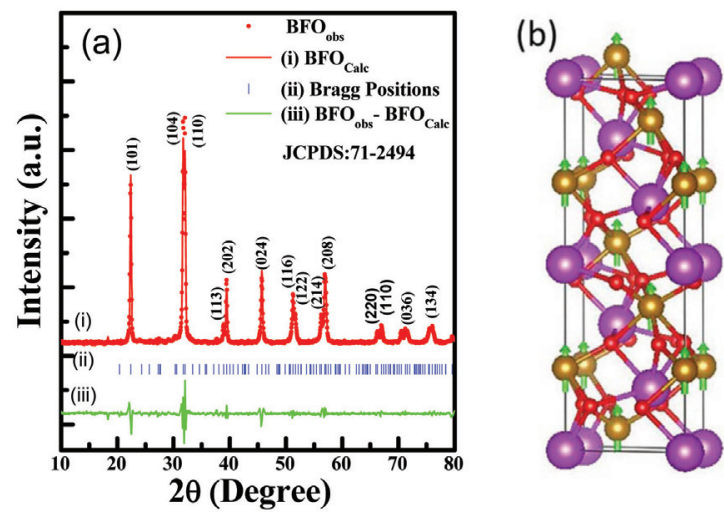

Fig. 1. (a) Powder X-ray diffraction pattern with refinement of as-synthesized BFO nanoparticles. (b) Crystal structure of BFO sample with ferromagnetic spin (Purple color sphere indicates $\mathrm{Bi}$ atom, golden color sphere is $\mathrm{Fe}$ atom, red color sphere shows $\mathrm{O}$ atom and green arrow indicates polarization direction).

by the Wien2k framework. The Hubbard corrected generalized gradient approximation $(\mathrm{GGA}+\mathrm{U})$ was used to define the electronic and thermoelectric properties. Here we treated the 94 valence electrons per unitcell. Moreover, muffin tin radius of $\mathrm{Bi}, \mathrm{Fe}$ and $\mathrm{O}$ fixed as 2.29, 1.87 and 1.61 respectively such a way that atoms not overlap each other. ${ }^{[9]}$ The wave function basis set were expand up to $R_{m t} \times K_{\max }$ as 7.0. Furrier expanded charge density fixed as $12\left(\mathrm{au}^{-1}\right)$. We used 500 $\mathrm{k}$-points to optimize the experimental obtained structure. To define the Electronic properties such as density of states and band structure, we performed $1000 \mathrm{k}$-point for irreducible brilluoin zones. The self-consistence total energy convergent accuracy secured to $10^{-6} \mathrm{Ry}$. Thermal conductivity and temperature dependent susceptibility features obtained from Boltztrap code. Here we treat the Fe-atom $3 \mathrm{~d}$-orbital as spin nature about to $4 \mathrm{eV} .{ }^{[10,11]}$ The energy cut-off set as $10^{-6}$ to separate the valance electron from core. To calculate optical properties we used 1000 $\mathrm{k}$-points,

\section{Result and Discussions}

The powder X-ray diffraction (XRD) pattern and crystal structure of as-synthesized pure $\mathrm{BiFeO}_{3}$ (BFO) sample is shown in Fig. 1. The diffraction features of the sample (Fig. 1 (a)) shows hexagonal structure with R3c space group. All the peaks well indexed and matches with the single phase $\mathrm{BFO}$ according to standard crystallographic data and it consistent to the JCPDS card No. 71-2494. ${ }^{[12]}$ The strong diffraction peaks of the sample (Fig. 1 (a)) suggest good crystalline nature. The 2 $\theta$ position at $31.723^{\circ}$ and $32.021^{\circ}$ are found to be highest intensity represent (104) and (110) plane. To further understand the crystal structure and lattice parameters, Rietveld refinement of the powder XRD pattern for as-synthesized BFO (Fig. 1 (a)) was performed using FullPROF Software. A third-order polynomial was employed to integrate the background while the peaks were fitted using a pseudo-Voigt function. ${ }^{[13]}$ The half width of the diffraction peaks as a function of $2 \theta$ was estimated by the Caglioti equation. It is to be noted that the simulated XRD pattern (red line $\mathrm{BFO}_{\text {calc }}$ ) is well matched with the experimental XRD peak (red dot $\mathrm{BFO}_{\text {obs }}$ ) as shown in Fig. 1 (a). Moreover, the lattice parameters of 5.633 and $13.933 \AA$ for a and c, respectively, were obtained from the refined structural parameters (Table 1). To demonstrate a successful refinement, the residuals (e.g., weighted profile residual $\left(\mathrm{R}_{\mathrm{wp}}\right)$, profile residual $\left(R_{p}\right)$, expected error residual $\left(R_{e}\right)$ and structure factor $\left(R_{f}\right)$ ), associated with the goodness of fit $\left(\mathrm{GOF}=\left(\mathrm{R}_{\mathrm{wp}} / \mathrm{R}_{\mathrm{e}}\right)\right)$ and $\operatorname{chi}^{2}\left(\chi^{2}\right)$, are also listed in Table 1. The low value of GOF and $\chi^{2}$ suggest 
(a)

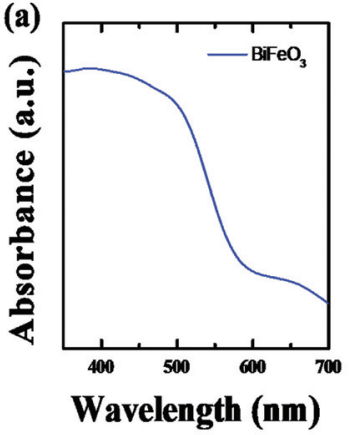

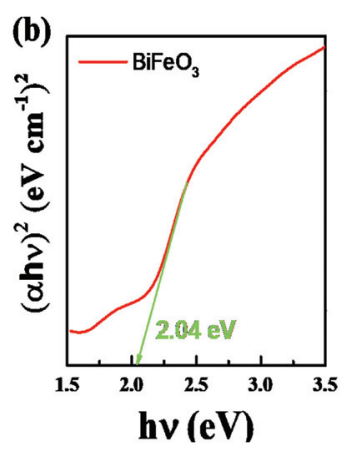

Fig. 2. (a) UV-vis diffuse reflectance absorption spectra of $\mathrm{BFO}$ and (b) analysis of UV-Vis measurement as a function of photon energy for $(\alpha h v)^{2}$.

the XRD pattern is well fitted to the hexagonal phase with R3C structure. Moreover, based on the refined parameters, the observed and calculated $2 \theta$ position along with the interplanner distance (d-values) is found to be little shifted, which may be ascribed to the difference in ionic radii. In addition, the crystal structure of as-synthesized BFO sample was visualized using the VESTA program (Fig. 1 (b)).

The electronic structure and bandgap of semiconductors is essentially linked with the optical absorption properties. The UV-VIS spectra of BFO for absorption from 400 to $700 \mathrm{~nm}$ at room temperature is shown in Fig. 2 (a). The sharp enhancement was observed in the UVVis spectra above the wavelength of $600 \mathrm{~nm}$, suggesting a considerable absorption of visible light. This suggests a potential use of $\mathrm{BFO}$ as a visible-light active photocatalyst. Bandgap of $\mathrm{BFO}$ can be estimated from the relation between the absorption coefficient $(\alpha)$ and incident photon energy (hv) near the band edge following, ${ }^{[14]}$

$$
\alpha h v=\mathrm{A}\left(h v-E_{g}\right)^{\mathrm{n} / 2}
$$

where $h$ is Plank constant, $v$ is light frequency,

Table 1. The Rietveld refined parameters and the comparison of observed and calculated $2 \theta$ values with respect to interplanner distance (d-value) for as-synthesized BFO.

\begin{tabular}{|c|c|c|c|c|c|c|c|c|c|c|}
\hline $\begin{array}{c}\text { Sample } \\
\text { notation }\end{array}$ & \multicolumn{2}{|c|}{ Residuals } & \multicolumn{2}{|c|}{$\begin{array}{c}\text { Lattice } \\
\text { parameters }\end{array}$} & $c / a$ & $\begin{array}{l}\text { Volume } \\
\left(\AA^{3}\right)\end{array}$ & $2 \theta_{\text {obs }}$ & $2 \theta_{\text {calc }}$ & $d_{o b s}$ & $d_{\text {cal }}$ \\
\hline \multirow{17}{*}{$\mathrm{BFO}$} & \multirow{3}{*}{$\mathrm{R}_{\mathrm{p}}$} & \multirow{3}{*}{19.4} & \multirow{8}{*}{$\mathrm{a}(\AA)$} & \multirow{8}{*}{5.633} & \multirow{17}{*}{2.473} & \multirow{17}{*}{382.853} & 22.403 & 22.400 & 3.9682 & 3.9653 \\
\hline & & & & & & & 27.372 & 27.298 & 2.8182 & 3.2557 \\
\hline & & & & & & & 31.723 & 31.729 & 2.7938 & 2.8184 \\
\hline & \multirow{3}{*}{$\mathrm{R}_{\mathrm{wp}}$} & \multirow{3}{*}{17.8} & & & & & 32.041 & 32.044 & 2.3910 & 2.7911 \\
\hline & & & & & & & 38.923 & 38.923 & 2.3112 & 2.3120 \\
\hline & & & & & & & 39.439 & 39.449 & 2.2844 & 2.2829 \\
\hline & \multirow{3}{*}{$\mathrm{R}_{\mathrm{e}}$} & \multirow{3}{*}{9.47} & & & & & 45.719 & 45.719 & 1.9841 & 1.9829 \\
\hline & & & & & & & 51.273 & 51.272 & 1.7808 & 1.7804 \\
\hline & & & \multirow{9}{*}{$c(\AA)$} & \multirow{9}{*}{13.933} & & & 51.647 & 51.696 & 1.7685 & 1.7684 \\
\hline & \multirow{3}{*}{$\mathrm{R}_{\mathrm{f}}$} & \multirow{3}{*}{0.718} & & & & & 56.335 & 56.322 & 1.6318 & 1.6318 \\
\hline & & & & & & & 56.965 & 56.919 & 1.6276 & 1.6152 \\
\hline & & & & & & & 66.272 & 66.286 & 1.4091 & 1.4092 \\
\hline & \multirow{3}{*}{ GOF } & \multirow{3}{*}{1.88} & & & & & 67.004 & 67.010 & 1.3969 & 1.3956 \\
\hline & & & & & & & 70.550 & 70.579 & 1.3330 & 1.3339 \\
\hline & & & & & & & 71.293 & 71.282 & 1.3227 & 1.3218 \\
\hline & \multirow{2}{*}{$\mathrm{Chi}^{2}$} & \multirow{2}{*}{3.541} & & & & & 75.561 & 75.534 & 1.2581 & 1.2573 \\
\hline & & & & & & & 76.038 & 76.048 & 1.2515 & 1.2506 \\
\hline
\end{tabular}




\section{특 집 므 G Thamizharasan, Eithiraj R.D, Enhbayar Enhtuwshin, So Jung Kim, Niroj Kumar Sahu, Arpan Kumar Nayak, HyukSu Han}
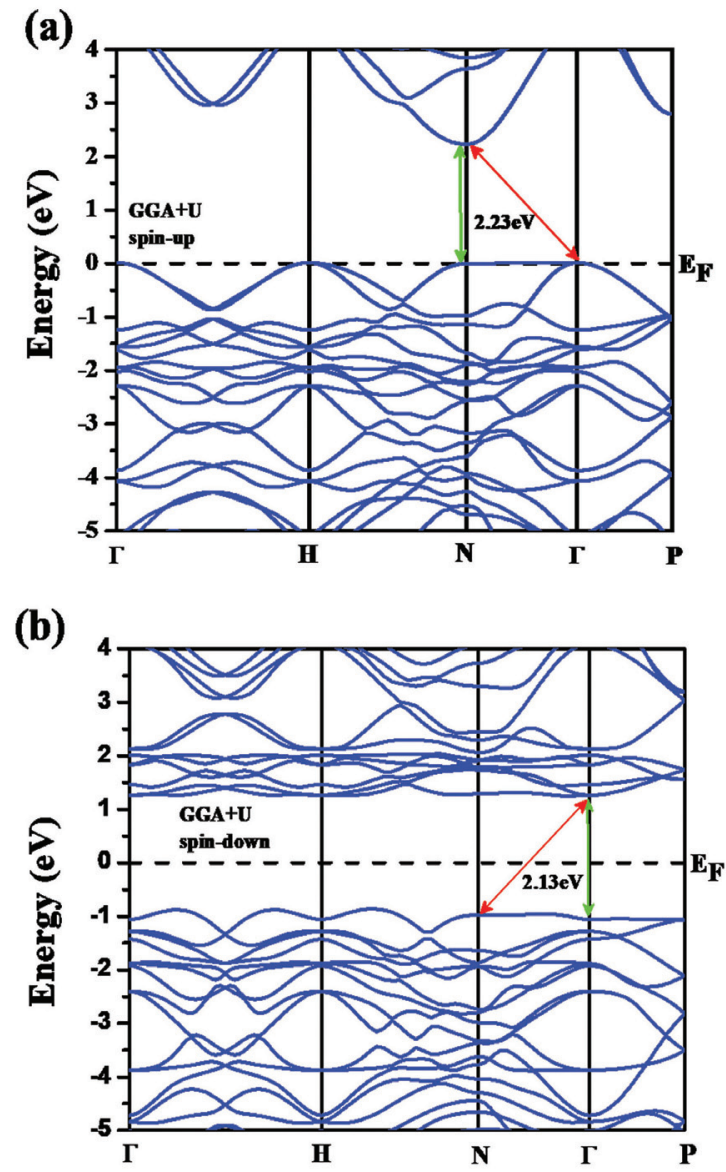

Fig. 3. Calcualted band structure of BFO using GGA+U method (a) sping up and (b) spin-down of Fe atoms.

and $E_{g}$ is a bandgap. $\alpha$ can be calculated from the experimental UV-Vis spectra. Given that BFO has a direct bandgap, the value of 1 can be used for $n$. ${ }^{[15]}$ Then, the bandgap of BFO can be obtained by extrapolating the linear plot of $(\alpha h v)^{2}$ vs. incident photon energy (hv). As can be seen in Fig. 2 (b), the direct bandgap of $\mathrm{BFO}$ was calculated as about $2.04 \mathrm{eV}$ which is comparable to the previously reported values. ${ }^{[16]}$ This reveals that $\mathrm{BFO}$ has a very narrow bandgap compared to other photocatalytic materials, highlighting its potential use for photoelectrochemical water splitting.

Computational calculations were employed to further study electronic band structure of $\mathrm{BFO}$. Electronic band structure of BFO using DFT with GGA+U method is shown in Fig. 3. Both of spin-up (Fig. 3 (a)) and spin-down (Fig. 3 (b)) states for Fe ion were considered for the calculations. The results indicate that $\mathrm{BFO}$ can have both of direct $(\mathrm{N}-\mathrm{N})$ and indirect $(\Gamma-\mathrm{N}$, very close to direct with only $0.001 \mathrm{eV}$ higher energy) bandgaps with values of 2.23 and $2.13 \mathrm{eV}$ for spin-up and spin-down states, respectively). The predicted indirect bandgap of $\mathrm{BFO}$, although very close to direct, is contradict with the experimental results. Notably, although an onset absorption starts below the bandgap in the absorption measurement (Fig. 2 (b)), this weak optical transition could be ignored for experimentally determining the bandgap. Thus, the absorption experimental data of $\mathrm{BFO}$ results in a direct bandgap for the most of case. ${ }^{[5]}$ Total density of states ( $t-D O S$ ) of BFO where the Fermi level is set as $0 \mathrm{eV}$ is presented in Fig. 4 (b). Notably, a weak ferromagnetic effect was clearly observed neat at the Fermi level as shown in the inset of Fig. 4 (a). Taking look into partial DOS ( $p$-DOS) plots of BFO, Bi and O have a symmetric p-DOS for spin-up and spin-down states (Fig. 4 (b)), however Fe shows unsymmetrical p-DOS for the two different spin states. The valence band (VB) of $\mathrm{BFO}$ is consisted of $\mathrm{Fe} 3 \mathrm{~d}, \mathrm{O} 2 \mathrm{p}$, and $\mathrm{Bi}$ $6 \mathrm{p}$ states. Additionally, the VB near at the Fermi level is mainly occupied by $\mathrm{Fe} 3 \mathrm{~d}$ and $\mathrm{O} 2 \mathrm{p}$ states indicating that a strong hybridization is occurred between $\mathrm{Fe}$ and $\mathrm{O}$ atoms. In the conduction band $(\mathrm{CB})$ of $\mathrm{BFO}$, it was found that Fe $3 \mathrm{~d}$ states predominantly occupy the $\mathrm{CB}$ in the range from 1 to $4 \mathrm{eV}$ and Bi $6 \mathrm{p}$ states mainly occupy above 4 $\mathrm{eV}$. Also, O 2p states are highly mixed with Fe 3d 

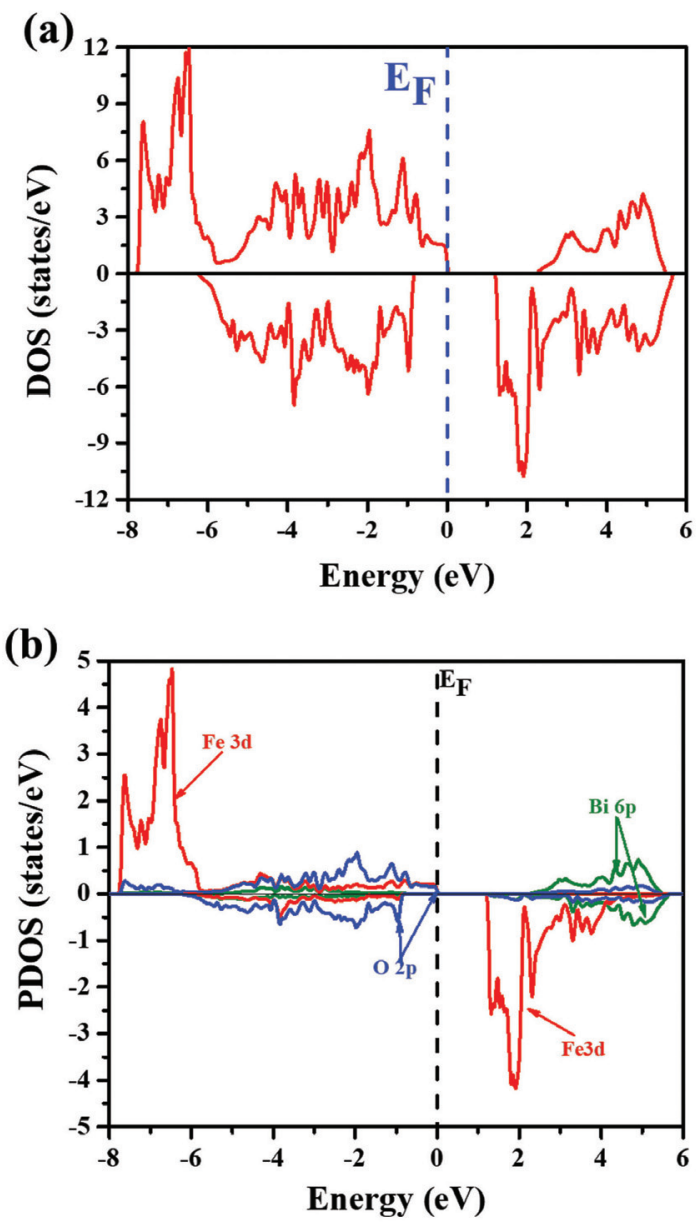

Fig. 4. Calculated (a) t-DOS and (b) p-DOS of BFO.

and $\mathrm{Bi} 6 \mathrm{p}$ sates in the $\mathrm{CB}$. Hence, the bandgap of $\mathrm{BFO}$ is constructed with $\mathrm{Fe} 3 \mathrm{~d}$ and $\mathrm{O} 2 \mathrm{p}$ states at the bottom of $\mathrm{CB}$ and the top of $\mathrm{VB}$, respectively. In regard with the strong hybridization between Fe $3 d$ and $\mathrm{O} 2 \mathrm{p}$ states in $\mathrm{BFO}$, charge transfer can occur from the occupied $\mathrm{O} 2 \mathrm{p}$ to the unoccupied Fe $3 d$ states or from VB to CB of the Fe 3d states through the $d-d$ transition. These two different electronic transition routes in $\mathrm{BFO}$ can effectively facilitate charge transfer of electron-hole pairs possibly boosting its photocatalytic activity.

A simulated complex dielectric function of $\mathrm{BFO}$ is shown in Fig. 5. Note that $\varepsilon_{2}$ (imaginary part of
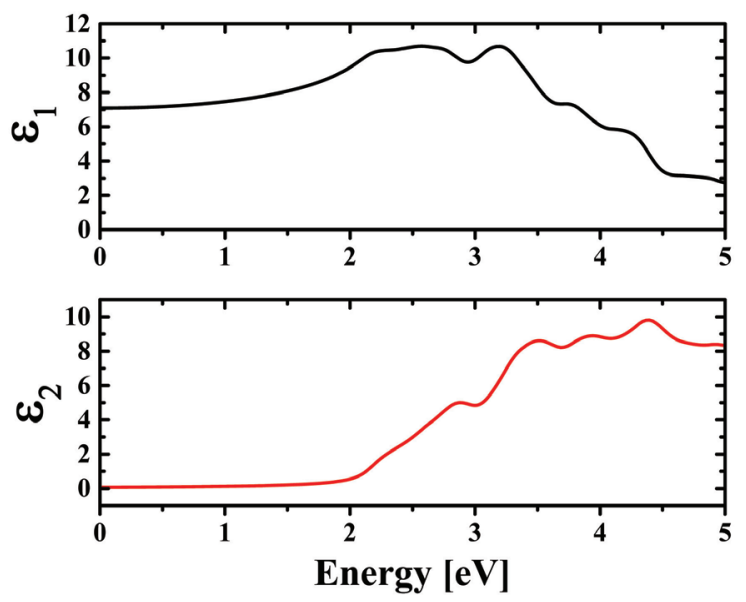

Fig. 5. Calculated complex dielectric function of BFO.

dielectric function) is essentially related with the electronic band structure where a peak in $\varepsilon_{2}$ vs. energy plot corresponds to a multiple direct or indirect interband transition. The $\varepsilon_{2}$ of $\mathrm{BFO}$ was started to increase approximately at $2.0 \mathrm{eV}$, where the first peak at $2.9 \mathrm{eV}$ can be ascribed to the transitions from $\mathrm{O} 2 \mathrm{p}$ in VB to Fe $2 \mathrm{~d}$ in $\mathrm{CB}$ or the $\mathrm{d}-\mathrm{d}-$ transition in Fe $3 d$ states. The peaks appeared at higher energy may be attributed to the transition from the occupied $\mathrm{O} 2 \mathrm{p}$ in VB to the unoccupied Fe $3 \mathrm{~d}$ or Bi $6 \mathrm{p}$ states with a higher energy states in $\mathrm{CB}$. Fig. 6 shows a simulation result on the absorption coefficient of $\mathrm{BFO}$ as a function of energy. It is found that the simulated absorption spectra of $\mathrm{BFO}$ gives a direct bandgap of $2.2 \mathrm{eV}$ which is well consistent with our experimental results.

\section{Conclusion}

In summary, we have studied the electronic band structure of $\mathrm{BFO}$, which was synthesized via a facile sol-gel method. Structural characterization was performed on the synthesized BFO powder by measuring the powder XRD pattern in combination 
with the Rietveld refinement. Electronic band structure of $\mathrm{BFO}$ was studied by experimentally measuring absorption spectra and computationally simulating band structure. The experimental as well as computational results reveal that $\mathrm{BFO}$ can have both of direct or indirect (close to direct) bandgaps with a value of around $2.0 \sim 2.2 \mathrm{eV}$. Also, the simulated electronic structure of $\mathrm{BFO}$ indicated that a weak ferromagnetic effect is existed in BFO. According to the detailed study on DOS plots, electronic transition in BFO can occur via two different routes such as the $\mathrm{O} 2 \mathrm{p}$ to $\mathrm{Fe}$ $3 d$ states or the $d-d$ transition in Fe $3 d$ states. The multiple electronic transition way in $\mathrm{BFO}$ with a narrow bandgap makes it as a promising photocatalytic material which can effectively absorb visible light.

\section{Data accessibility}

There are no additional data to accompany this manuscript. All relevant datasets are within the main body of the manuscript. All protocols and software used are stated fully in the methodology section. There are no coding lists for this research.

\section{Author contributions}

H.H. and A.K.N. conceived the project; H.H. and A.K.N designed the experiments; H.H. and A.K.N wrote the manuscript; G.T., E.R.D., N.K.S, E.E. performed the detailed analysis on the data; G.T., E.R.D., N.K.S, S.J.K. performed the detailed analysis on structural data of the samples; All authors participated in analyzing the data and commented on the manuscript; All authors gave final approval for publication.

\section{Competing interest}

There are no competing interests for this manuscript.

\section{Research ethics}

No research which needs a local ethic approval is included in this work.

\section{Animal ethics}

No animal related experiments are included in this work

\section{Permission to carry out fieldwork}

No field works are included in this work.

\section{Funding}

This research was supported by Basic Science Research Program through the National Research Foundation of Korea (NRF) funded by the Ministry of Science, ICT and Future Planning (2018R1D1A1A02085938).

\section{Acknowledgments}

Authors thank for Dr. H. Kim for valuable discussion on the manuscript.

\section{REFERENCES}

1. Yin ZY, Wang Z, Du YP, Qi XY, Huang YZ, Xue C, Zhang H. Full solution-processed synthesis of all metal oxide-based tree-like heterostructures on fluorinedoped tin oxide for water splitting. Adv Mater. 24(39), 
5374-5378 (2012). doi:10.1002/adma.201201474

2. Reddy CV, Reddy KR, Shetti NP, Shim J, Aminabhavi TM, Dionysiou DD. Hetero-nanostructured metal oxide-based hybrid photocatalysts for enhanced photoelectrochemical water splitting - a review. Int J Hydrogen Energ. 45(36), 18331-18347 (2020). doi:10.1016/j.ijhydene.2019.02.109

3. Chu YH, Martin LW, Holcomb MB, Gajek M, Han SJ, He Q, Balke N, Yang CH, Lee D, Hu W, Zhan Q, Yang PL, Fraile-Rodriguez A, Scholl A, Wang SX, Ramesh R. Electric-field control of local ferromagnetism using a magnetoelectric multiferroic. Nat Mater. 7(6), 478482 (2008). doi:10.1038/nmat2184

4. Wang J, Neaton JB, Zheng H, Nagarajan V, Ogale SB, Liu B, Viehland D, Vaithyanathan V, Schlom DG, Waghmare UV, Spaldin NA, Rabe KM, Wuttig M, Ramesh R. Epitaxial bifeo3 multiferroic thin film heterostructures. Science. 299(5613), 1719-1722 (2003). doi:DOI 10.1126/science.1080615

5. Gao F, Yuan Y, Wang KF, Chen XY, Chen F, Liu JM. Preparation and photoabsorption characterization of bifeo3 nanowires. Appl Phys Lett. 89(10), (2006). doi:Artn 102506 10.1063/1.2345825

6. McDonnell KA, Wadnerkar N, English NJ, Rahman M, Dowling D. Photo-active and optical properties of bismuth ferrite (bifeo3): An experimental and theoretical study. Chem Phys Lett. 572(78-84 (2013). doi:10.1016/j.cplett.2013.04.024

7. Ji W, Yao K, Liang YC. Bulk photovoltaic effect at visible wavelength in epitaxial ferroelectric bifeo3 thin films. Adv Mater. 22(15), 1763-+ (2010). doi:10.1002/ adma.200902985

8. Wang H, Zheng Y, Cai MQ, Huang H, Chan H.L.W, First-principles study on the electronic and optical properties of BiFeO3. Solid State Communications, 149, 641-644 (2009). doi:10.1016/j.ssc.2009.01.023

9. Yaakob MK, Taib MFM, Deni MSM, Yahya MZA. Ab initio studies on the structural and electronic properties of bismuth ferrite based on ferroelectric hexagonal phase and paraelectric orthorhombic phase. Integr
Ferroelectr. 155(1), 134-142 (2014). doi:10.1080/1058 4587.2014.905306

10. Singh S, Haraldsen JT, Xiong J, Choi EM, Lu P, Yi D, Wen XD, Liu J, Wang H, Bi Z, Yu P, Fitzsimmons MR, MacManus-Driscoll JL, Ramesh R, Balatsky AV, Zhu JX, Jia QX. Induced magnetization in la0.7sr0.3mno3/bifeo3 superlattices. Phys Rev Lett. 113(4), (2014). doi:ARTN 047204 11.1103/ PhysRevLett.113.047204

10. Shenton JK, Bowler DR, Cheah WL. Effects of the hubbard $\mathrm{u}$ on density functional-based predictions of bifeo3 properties. J Phys-Condens Mat. 29(44), (2017). doi:ARTN 445501 10.1088/1361-648X/ aa8935

12. Yu XL, An XQ. Enhanced magnetic and optical properties of pure and ( $\mathrm{mn}, \mathrm{sr})$ doped bifeo3 nanocrystals. Solid State Commun. 149(17-18), 711714 (2009). doi:10.1016/j.ssc.2009.02.010

13. Kumar A, Varshney D. Crystal structure refinement of bil-xndxofeo3 multiferroic by the rietveld method. Ceram Int. 38(5), 3935-3942 (2012). doi:10.1016/ j.ceramint.2012.01.046

14. Podraza NJ, Qiu W, Hinojosa BB, Xu HX, Motyka MA, Phillpot SR, Baciak JE, Trolier-McKinstry S, Nino JC. Band gap and structure of single crystal bii3: Resolving discrepancies in literature. J Appl Phys. 114(3), (2013). doi:Artn 033110 10.1063/1.4813486

15. Hauser AJ, Zhang J, Mier L, Ricciardo RA, Woodward PM, Gustafson TL, Brillson LJ, Yang FY. Characterization of electronic structure and defect states of thin epitaxial bifeo(3) films by uv-visible absorption and cathodoluminescence spectroscopies. Appl Phys Lett. 92(22), (2008). doi:Artn 222901 10.1063/1.2939101

16. Takahashi K, Kida N, Tonouchi M. Terahertz radiation by an ultrafast spontaneous polarization modulation of multiferroic bifeo3 thin films. Phys Rev Lett. 96(11), (2006). doi:ARTN 117402 10.1103/ PhysRevLett.96.117402 